\title{
THE POTENTIAL DIFFERENCES BETWEEN MULTIPLE CENTRAL TERMINALS EACH CONNECTED TO A SEPARATE SET OF LIMB ELECTRODES
}

\author{
Morley J. Kert, M.D.,* Los Angeles, Calif., AND \\ J. Marion Bryant, M.D., Ann Arbor, Mich.
}

IN A recent publication Groedel ${ }^{1}$ reports that he has found significantly large potential differences between three central terminals each connected to a separate set of three limb electrodes. He does not mention the size of the resistances between the limb electrodes and the central terminals. One central terminal was attached to electrodes near the junctions of the extremities with the trunk, one to electrodes near their distal ends, and the third to electrodes near the second joints. The largest potential difference observed amounted to approximately 0.5 millivolt. It was stated that "a final electrophysical explanation" could not be given. The conclusions, however, were "that at the central terminal there is a considerable potential, which is neither zero nor approximately zero," and "that the central terminal offers no practical advantage for its further use in obtaining so-called unipolar chest leads."

It is not our purpose here to attempt either to prove or to disprove that the potential variations of a central terminal connected to the limb electrodes through large resistances are negligible, but rather to explore the situation which exists when several central terminals are joined to electrodes on the limbs in the manner specified.

\section{METHODS}

Two metal electrodes approximately $1.0 \mathrm{~cm}$. in diameter were placed in the midsternal line and connected to the output terminals of a beat-frequency oscillator. In this way a low-frequency (25 cycles per second) alternating current of 0.35 to 0.5 milliampere was introduced into the chest of a normal subject. The strength of the electrical field thus set up in the body could be varied either by changing the size of the current or by changing the distance between the input electrodes. Our purpose in creating an artificial electrical field, instead of studying that associated with the heart beat, was to place the intensity of the field under our control and to substitute a simple wave form for the complicated electrocardiographic deflections.

Five German silver electrodes of the pattern commonly used in electrocardiographic studies were placed on each of the three extremities from which the

From the Department of Internal Medicine, University of Michigan Medical School, The observations upon which this article is based were made with the aid of grants to Dr. Frank N. Wilson from the Horace H. Rackham School of Graduate Studies and the S. S. Kresge Foundation.

*Aided by a fellowship from the Dazian Foundation for Medical Research. 
standard limb leads are taken. The arrangement of these electrodes is shown in the diagram reproduced in Fig. 1 . The electrodes labeled 1,2 , and 3 were approximately two inches apart and those labeled $A$ and $B$, approximately five inches apart. Two additional electrodes, not shown in Fig. 1, were placed at the humeral attachments of the deltoid muscles for the purpose of taking standard Lead I. This lead was taken simultaneously with each of the other leads employed. All records were taken with the Sanborn Tri-beam electrocardiograph. Each

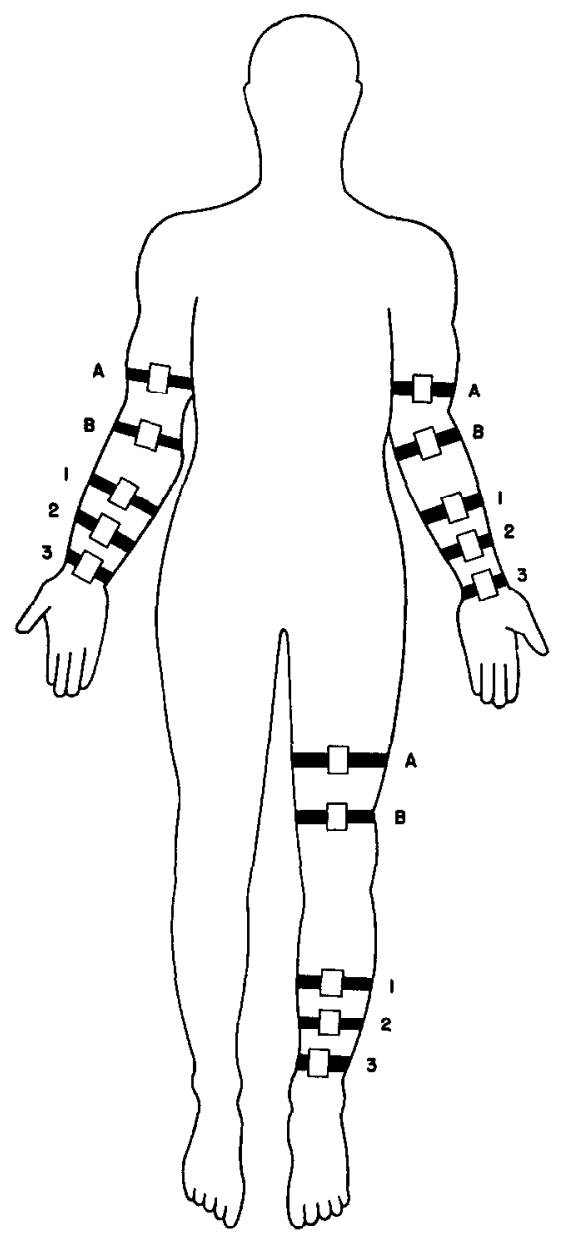

Fig. 1.-Diagrammatic representation of the locations of the electrodes in Experiments 1 and 2. Leads from Electrode $A$ to Electrode $B$ were used to estimate changes in the current flowing along the extremity produced by connecting the distal electrodes to one or more central terminals. Numbers 1,2, and 3 indicate the positions of the limb electrodes to which the central terminals were attached.

central terminal used was connected to the Number 1, the Number 2, or the Number 3 electrodes. The more proximal electrodes, $A$ and $B$, were used to obtain information concerning the flow of current along each extremity when one or more of the distal electrodes was connected to a central terminal. In Figs. 2 and 3 the $A$ to $B$ lead from the right arm is labeled $R$; that from the 
left arm, $L$; and that from the left leg, $F$. When more than one central terminal was connected, the potentials of the various central terminals were compared by leading from one to the other.

\section{DESCRIPTION OF EXPERIMENTS}

Experiment 1.-In this instance the current and the distance between the input electrodes were adjusted in such a way as to yield deflections in Leads II and III of the maximum size that could be conveniently recorded simultaneously with Lead I on bromide paper $6.0 \mathrm{~cm}$. in width. The electrocardiograph was operated at its normal sensitivity. The input current was 0.35 milliampere and it was maintained at this value with the aid of a milliammeter. All deflections were measured to the nearest 0.5 millimeter. Leads from the $A$ to the $B$ electrode on each extremity were taken before and after a central terminal was connected directly, that is, without resistances, to one of the sets of distal electrodes, and after first one, then a second, and then a third central terminal was connected through $5,000 \mathrm{ohm}$ resistors in successive steps. Finally, the potential differences between the central terminals connected through resistors were recorded; that is, the single potential difference when only two were connected, and the three potential differences between the different pairs when three were connected.

Strips of the tracings obtained are reproduced in Fig. 2. These were cut in such a way as to avoid sections where the sine waves were distorted by the subject's electrocardiogram. The standard limb leads show very large deflections in Leads II and III and these are out of phase with the small oscillations in Lead I. Consequently, the algebraic sum of the deflections in Leads I and III is 43 millimeters. The deflections in Lead II measure 40 millimeters. Before any of the distal electrodes $(1,2$, and 3$)$ were connected through a central terminal the oscillations in the leads from the $A$ to the $B$ electrode on each of the three extremities (labeled $R, L$, and $F$ ) were so small as to be barely visible. At this time, therefore, the currents flowing through the internal tissues of the extremities toward or away from the trunk were of negligible magnitude. When a central terminal was joined to a set of distal electrodes without intervening resistances, the deflections in these same leads approached those of Lead I in size. On the other hand, they were not much larger after than before a central terminal was attached to the same set of limb electrodes through $5,000 \mathrm{ohm}$ resistors.

They increased in amplitude when a second, and again when a third central terminal of the same kind was connected, but the currents flowing through the limbs were only about two-thirds as large after the connection of three central terminals through resistors as they were after the connection of one terminal without resistors. When two central terminals were connected to the extremities through resistors, the difference in potential between them was small. When three central terminals were connected through resistors, the differences in potential between the different pairs were somewhat larger and not all equal. The largest oscillations recorded by leading from one terminal to another are about $1.0 \mathrm{~mm}$. in amplitude. 


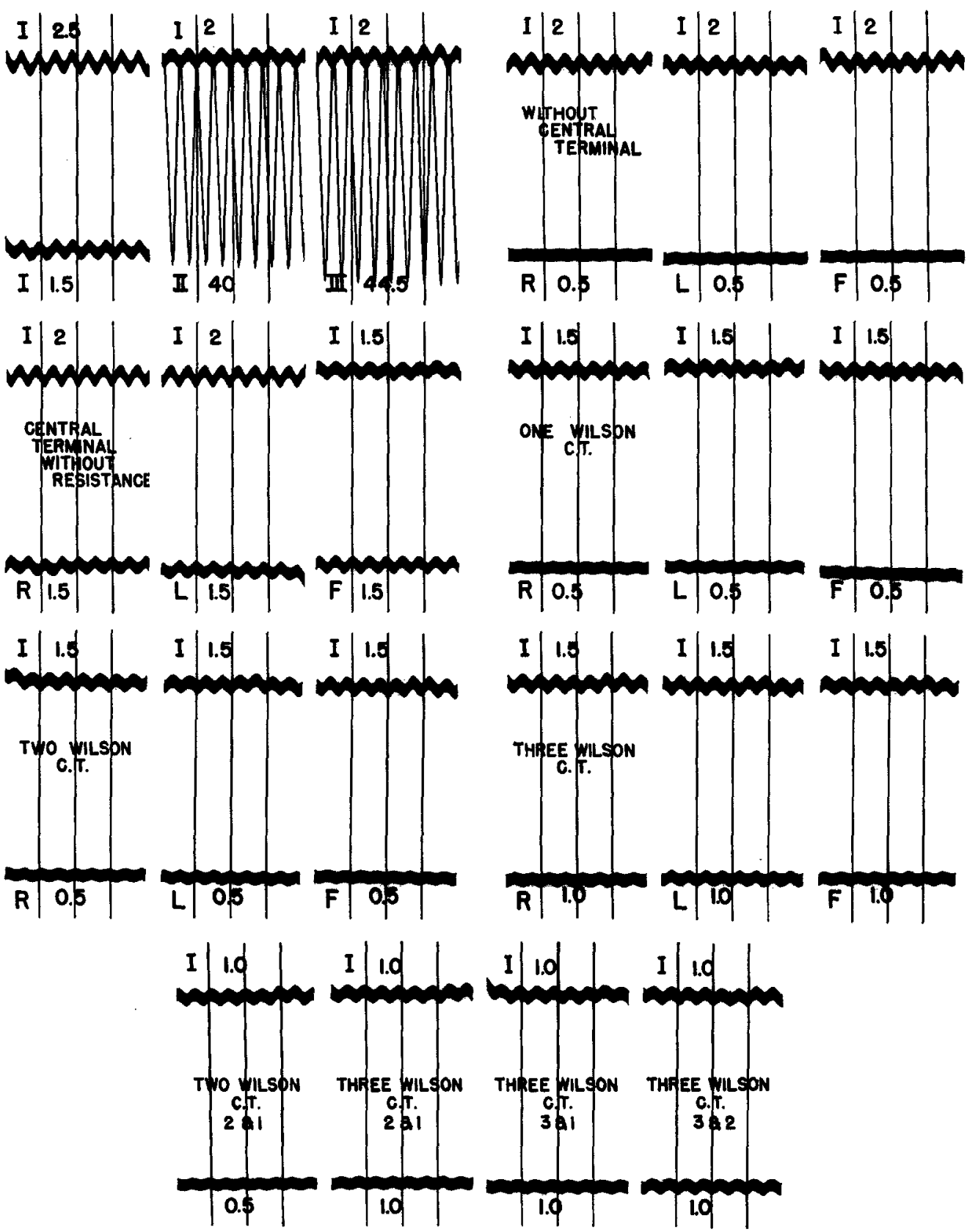

Fig. 2.-Experiment 1. Records of potential differences produced by introducing a small 25 cycle current into the body of a normal subject. Leads $R, L$, and $F$ are leads from the $A$ to the $B$ electrodes on the right arm, left arm, and left leg, respectively. The leads at the bottom of the figure show the differences in potential between the various central terminals. Lead I was taken simultaneously with each of the other leads used. Arabic numerals give the amplitudes of the deflections in millimeters. The electrocardiograph was used at its normal sensitivity. 
Experiment 2.-In order to obtain larger deflections a second experiment was performed in which the maximal current ( 0.5 milliampere) which the oscillator was capable of producing was employed. At the same time, the distance between the input electrodes was made as large as possible so as to give the electrical moment, which is measured by the product of the current and the distance between the electrodes, the greatest value attainable. In addition, the recorls were taken with the electrocardiograph operating at double its normal sensitivity. Under these circumstances the potential variations of the extremities were naturally of such great magnitude that it was impossible to record the deflections in Leads II and III. The potential differences between the Electrodes $A$ and $B$ and between the central terminals were recorded exactly as in Experiment 1.

The tracings obtained are reproduced in Fig. 3. Before any of the distal electrodes were joined to a central terminal the deflections in Leads $R, L$, and $F$, which are a measure of the currents flowing in the extremities, were slightly larger than those recorded in our first experiment under the same circumstances, but still extremely small. They became very large, however, when a central terminal was connected, without intervening resistors, to the limb electrodes of Set 1. The increased flow of current along the limbs was accompanied by a 50 per cent increase in the voltage of the deflections of Lead I. It will be noted that the large current in the leg $(F)$ was 180 degrees out of phase, while the cur. rents in the two arms ( $R$ and $L$ ) were in phase, with the potential differences which this lead recorded. Since the line joining the input electrodes was nearly parallel to the long axis of the trunk, the potential of the left leg was positive when the potentials of the two arms were negative, and vice versa. Consequently, the leg current was flowing toward the central terminal when the arm currents were flowing away from it, and conversely. It should be noted that the relative amplitude of the oscillations in Leads $\mathrm{R}$ and $\mathrm{L}$ measures the relative magnitude of the $I R$ drops between the $A$ and $B$ electrodes on the two arms and not necessarily the relative magnitude of the currents flowing in these extremities. There is, however, little question that in this particular instance the current in the left arm was considerably larger than that in the right; this was the cause of the increased voltage of the deflections in Lead $\mathrm{I}$.

When a central terminal with resistances of $5,000 \mathrm{ohms}$ was substituted for the central terminal without resistances, the deflections in Lead I decreased to their original size; the amplitude of the oscillations in Lead $\mathrm{R}$ fell from 6.0 to $3.0 \mathrm{~mm}$.; that of the oscillations in Lead L, from 16 to $3 \mathrm{~mm}$.; and that of the oscillations in Lead F, from 30 to 8 millimeters. When a second, and then a third, central terminal with resistances were connected, these oscillations became larger again, particularly in Leads $R$ and $F$. The failure of the current in the left arm to increase more than it did when the second and third terminals were added is rather surprising; it may be that the resistances of the areas of skin beneath the Number 2 and Number 3 electrodes on the left arm were large in comparison with the skin resistances beneath the corresponding electrodes on the other extremities. It will be noted that the disproportionate increase in the 

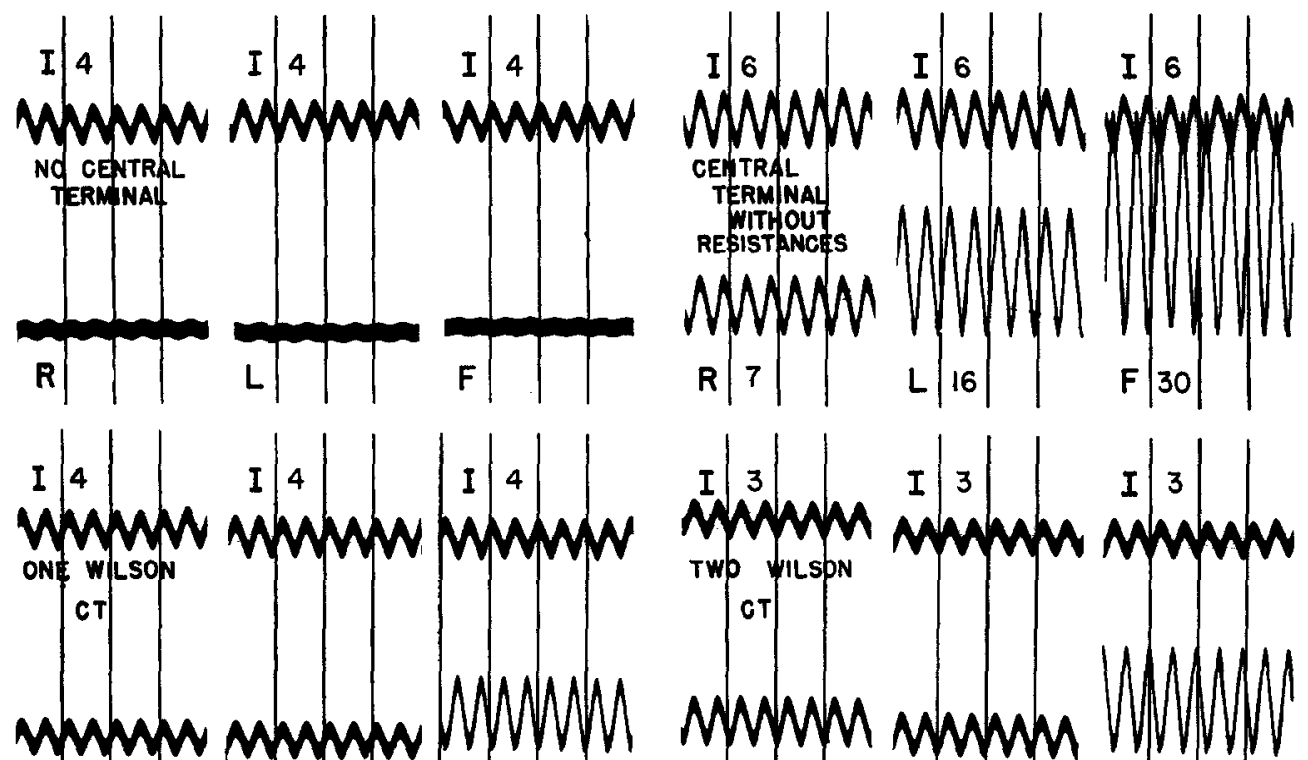

R 3

L. 3

$\mathbf{F}|8|$

MWMM

TW6 UILSpN

Mumm $A$
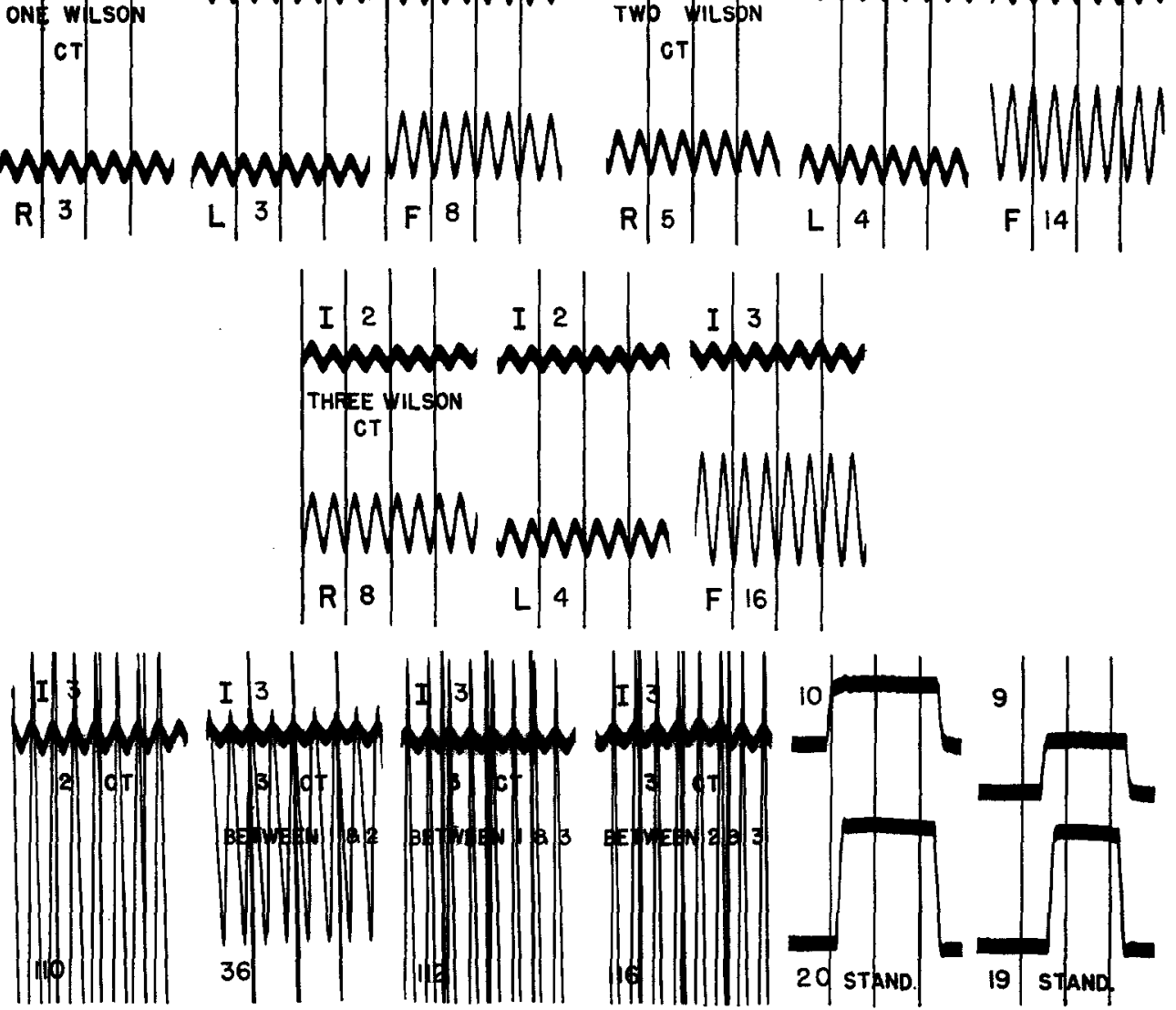

Fig. 3.- Experiment 2. The leads are the same as those shown in Fig. 2. The current and the distance between the input electrodes were adjusted to give the maximal electrical moment attainable. The lower beam was standardized at double the normal sensitivity. Arabic numerals give the amplitude of the deflections in millimeters. The last two strips show standardization before and after the experiment and demonstrate a very slight change in the sensitivity of the electrocardiograph. 
current in the right arm as compared to that in the left was accompanied by a reduction in the size of the deflections in Lead I.

All of the potential differences between the central terminals, with one exception, were so large that they could not be satisfactorily recorded. The last two strips of record in Fig. 3 show the effect of throwing a millivolt into the electrocardiographic circuit at the beginning of the experiment, and again at the end. The sensitivity of the instrument decreased slightly during the interval which elapsed between the two tests.

\section{DISCUSSION}

It is evident that the phenomena described were dependent, in one way or another, upon the absolute and relative magnitudes of the resistances, or other parameters, of the circuits established by connecting one or more central terminals to the electrodes on the limbs. The circuit elements referred to are indicated in the diagram reproduced in Fig. 4. The five electrodes, $A, B, 1,2$, and 3, on each of two extremities are shown, together with the resistances, $R_{\mathrm{a}}, R_{\mathrm{b}}, R_{1}$, and so forth, between them and the internal tissues. The resistances of the internal tissues of the segments of the extremities between the various electrodes and between the $A$ electrodes and the poles of the battery $E_{1}$, which represents the open-circuit potential difference between the two extremities, have been assigned the symbols $r_{\mathrm{a}}, r_{\mathrm{b}}, r_{\mathrm{s}}$, and so forth. To avoid complications the third extremity is not included in the diagram. When it is necessary to distinguish between the circuit elements of one extremity and those of another, we shall use unprimed symbols when referring to circuit elements associated with the right arm, primed symbols for the corresponding elements associated with the left arm, and double-primed symbols for those associated with the left leg. The symbols $A, A^{\prime}$, and $A^{\prime \prime}$, for example, refer to the $A$ electrodes on the right arm, left arm, and left leg, respectively. For the equal resistances in the branches of the central terminal we shall use the symbol $R$.

The circuit diagram of Fig. 4 is very much like that of a Wheatstone bridge, but has three branches connected in parallel instead of only two. These are the branches in which the central terminals lie. It is clear that the voltage drop in each of them is equal to the difference in potential between the nodes $X$ and $X^{\prime}$. The potential of each of the central terminals, with reference to that of either of these nodes, is determined by the ratio of the two resistances (or sums of resistances) which separates the one from the other. The potential of the terminal connected to the two Number 1 electrodes is, then, determined by the ratio $\left(R+R_{1}\right):\left(R+R^{\prime}{ }_{1}\right)$; that of the terminal connected to the Number 2 electrodes, by the ratio $\left(R+R_{2}+r_{\mathrm{d}}\right):\left(R+R^{\prime}{ }_{2}+r_{\mathrm{d}}^{\prime}\right)$; and that of the terminal connected to the Number 3 electrodes, by the ratio $\left(R+R_{3}+r_{0}+r_{\mathrm{d}}\right):\left(R+R^{\prime}{ }_{3}+r^{\prime}{ }_{0}+r_{\mathrm{d}}^{\prime}\right)$. When these three ratios are equal, the three terminals are necessarily always at the same potential, and when any two of them are equal, the potentials of the corresponding terminals are equal. If the two equal resistances $R$ are very large in comparison with the differences in magnitude between the members of each of the other pairs of resistances involved, the differences in potential 
between the three central terminals will be negligible. The members of the pairs of skin resistances, $R_{1}, R_{1}^{\prime} ; R_{2}, R_{2}^{\prime}$; and $R_{3}, R_{3}^{\prime}$, are those most likely to be unequal, for the skin resistance is usually high in comparison with the resistance of the internal tissues and is to a large extent dependent upon the technique used

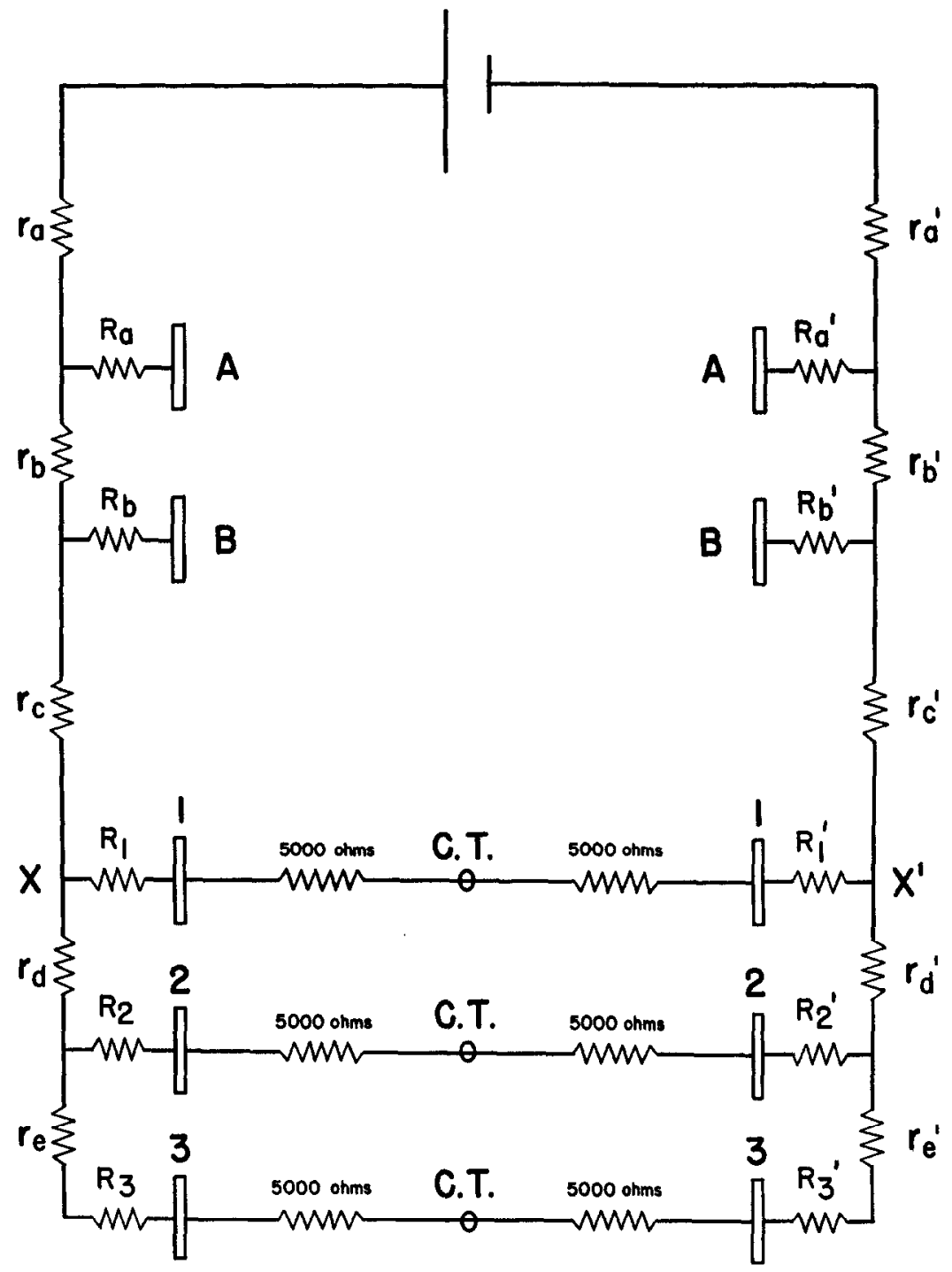

Fig. 4.-Schematic representation of the electrical circuits established by connecting three central terminals to separate sets of limb electrodes on two extremities. $r$, resistance of internal tissues; $R$, resistance of skin beneath electrode; $C T$, central terminal; $A$ and $B$, electrodes used to estimate changes in current flowing along extremity; 1,2 , and 3 , sets of Jimb electrodes attached to the three central terminals. For the sake of simplicity the third extremily is not shown in the diagram. See text for further details.

in attaching the electrodes to it. It would, of course, be possible to measure the resistances between the limb electrodes of each set and then equalize them by adding the appropriate resistances to the arms of each of the central terminals. 
This procedure would insure that all of the central terminals would be at the same potential. ${ }^{2}$

When each of the different central terminals is connected to three limb elec. trodes instead of two the situation is considerably more complicated, but the principles involved are the same. There are then three nodes, $X, X^{\prime}$, and $X^{\prime \prime}$. and the potential of each terminal with respect to them is determined by two ratios. In the case of the terminal connected to the Number 1 electrodes, these ratios are defined by the expression $\left(R+R_{1}\right):\left(R+R^{\prime}{ }_{1}\right):\left(R+R^{\prime \prime}{ }_{1}\right)$ The corresponding ratios for the other terminals are the following:

$$
\begin{aligned}
& \left(R+R_{2}+r_{\mathrm{d}}\right):\left(R+R_{2}+r^{\prime}{ }_{\mathrm{d}}\right):\left(R+R^{\prime \prime}{ }_{2}+r^{\prime \prime}{ }_{\mathrm{d}}\right) \text { and } \\
& \left(R+R_{3}+r_{\mathrm{d}}+r_{\mathrm{e}}\right):\left(R+R_{3}+r_{\mathrm{d}}+r_{\mathrm{e}}\right):\left(R+R^{\prime \prime}{ }_{3}+r^{\prime \prime}{ }_{\mathrm{d}}+r^{\prime \prime}{ }_{\mathrm{e}}\right) .
\end{aligned}
$$

If these ratios are equal the potentials of all three terminals must be the same.

The Leads $\mathrm{R}, \mathrm{L}$, and $\mathrm{F}$ in our experiments recorded the voltage drops across the resistances $r_{\mathrm{b}}, r_{\mathrm{b}}^{\prime}$, and $r^{\prime \prime}{ }_{\mathrm{b}}$, respectively. Consequently, the deflection in each of these leads represents the product of the corresponding resistance and the current flowing through it. Since the resistances involved are those of the internal tissues, it may be assumed that they did not vary. The currents. on the other hand, were determined by the ratios of the voltages acting in the respective circuits to the total resistances in these circuits. When the resistances between the limb electrodes are equal the equations which define the currents in the branches of the central terminal, and therefore in the extremities to which they are connected, are of the following type:

$$
i_{\mathrm{F}}=\frac{E_{2}+E_{3}}{R_{\mathrm{T}}+3 R_{\mathrm{F}}+3 R}
$$

Here $i_{\mathrm{F}}$ is the current in the left leg; $E_{2}$ and $E_{3}$ are the open-circuit voltages in Leads II and III, respectively; $R_{\mathrm{T}}$ is the resistance of the trunk; $R_{\mathrm{F}}$ is the total resistance of the left leg, and therefore represents the sum of the skin resistance and the resistance of the inner tissues; and $R$ is the value of the equal resistances in the branches of the central terminal.

It is obvious that when $R$ is large the currents in the extremities are small. When it is very large in comparison with the resistances in the limb leads, each of which is the sum of the resistances of two extremities plus the resistance of the trunk, the current in a given limb will be strictly proportional to the algebraic sum of the open-circuit voltages in the two standard limb leads in which that limb is attached to the electrocardiographic terminals. In this case inequalities of the skin resistances will have no significant effect upon the potential of the central terminal. When $R$ is small the opposite will be the case. The flow of current through the extremities will be large, and this will not only increase the effects in question but may give rise to others due to polarization in the skin. What may happen when the resistances in the arms of the central terminal are dispensed with is, therefore, unpredictable. Connecting three central terminals to separate sets of electrodes may be expected to have about the same effect upon the size of the currents in the limbs as that produced by reducing the magnitude of resistances in the arms of the central terminal by two-thirds. 


\section{CONCLUSIONS}

It is true that, under certain circumstances, multiple central terminals each connected to a separate set of limb electrodes are not at the same potential. The differences in potential between them are due to inequalities in the resistances between the limb electrodes to which they are attached, and these in turn are probably due chiefly to inequalities in the resistances of the areas of skin beneath these electrodes.

These differences in potential will not occur if the resistances between the limb electrodes are measured and equalized, or if the resistances in the arms of the central terminals are sufficiently large.

The phenomena in question do not have an important bearing upon the validity of the Einthoven triangle or upon the usefulness of the central terminal as a reference electrode. They do indicate that the resistances in the arms of the central terminal should be as large as practicable.

\section{REFERENCES}

1. Groedel, F. M.: Investigations on the Potential Fluctuations Existing at the Central Terminal Electrode, Exper. Med. \& Surg. 5:75, 1947.

2. Bryant, J. M., Johnston, F. D., and Wilson, F. N.: Unipolar Electrocardiographic Leads. Effects Produced by Eliminating the Resistors Between the Limb Electrodes and the Central Terminal, Am. Heart J. 37:321, 1949. 